\title{
Year in review in journal of clinical monitoring and computing 2014: cardiovascular and hemodynamic monitoring
}

\author{
Karim Bendjelid · Steffen Rex • Thomas Scheeren • \\ Lester Critchley
}

Received: 13 February 2015/Accepted: 13 February 2015/Published online: 5 March 2015

(c) Springer Science+Business Media New York 2015

Hemodynamic instability is a common sign in critically ill patients and its importance has been increasingly recognized during the last 20 years. Indeed, It is now appreciated that an adequate hemodynamic monitoring associated to reactive vigorous therapy is able to decrease the present ominous prognostic sign. In this regard, the Journal of Clinical Monitoring and Computing (JCMC) has become an ideal platform for publishing cardiovascular and hemodynamic monitoring-related research, as reflected by an increasing number of articles related to this topic and published in the recent years. To highlight this new progress, the journal decided to print a review on some papers published last year in the JCMC and related to cardiac output monitoring and cardiovascular functions.

\section{Evaluation of haemodynamic alterations and fluid responsiveness}

In the April issue, Yi Jo and colleagues investigated the big issue of hypotension associated with the beach chair

\footnotetext{
K. Bendjelid ( $\square$ )

Department of Anaesthesiology and Intensive Care, Geneva University Hospitals, Geneva, Switzerland

e-mail: Karim.Bendjelid@hcuge.ch

S. Rex

Department of Anesthesiology, University Hospitals Leuven, Herestraat 49, 3000 Louvain, Belgium

T. Scheeren

Department of Anaesthesiology, University Medical Center Groningen, University of Groningen, Groningen,

The Netherlands

L. Critchley

The Chinese University of Hong Kong, Hong Kong, China
}

position (BCP) during general anesthesia in forty healthy adult patients undergoing elective arthroscopic shoulder surgery [1]. Indeed, although fluid preloading with crystalloid or colloid fluids may alleviate the hemodynamic consequences associated with the BCP during general anesthesia, the present fluid therapy may possibly lead to volume overload. In this regard, the authors investigated whether hemodynamic variables obtained using the FloTrac/Vigileo system, including mean arterial pressure and stroke volume variations (SVV) could predict hypotension in mechanically ventilated patients undergoing BCP [1]. Hypotension related to the BCP posture occurred in $37.5 \%$ of patients in this study and the authors found that in contrast to post-induction value of SVV in mechanically ventilated state, pre-induction SVV cannot predict hypotension associated with the BCP in this study [1]. These results are related to the fact that dynamic indices are wellknown to predict fluid responsiveness only in controlled mechanically ventilated patients as they strongly depend on respiratory status. Interestingly, in the present study, preinduction values of $\mathrm{CI}$ and SVI before adopting the $\mathrm{BCP}$ have been also found to be good predictors of hypotension associated to the posture. And as highlighted by the authors, these findings suggest that not only preload but also preoperative cardiac performances might be the important factors for the development of hypotension after a repositioning supine to the sitting during general anesthesia.

Functional hemodynamic monitoring has gained increasing popularity in the hemodynamic management of critically ill patients. It has been repeatedly demonstrated that-in contrast to static preload parameters (such as filling pressures or volumetric variables)—only dynamic preload indicators (mainly derived from respiratory variations of arterial pressure) can reliably predict fluid responsiveness [2]. However, for the intraoperative period, it 
has recently been reported that due to the prevalence of these limitations, monitoring of ventilation-induced pressure variations is only applicable in approximately $40 \%$ of all patients undergoing surgery [3]. For patients being treated on an intensive care unit (ICU), no data have been available so far regarding the applicability of functional hemodynamic monitoring. In the issue of June, Benes et al. retrospectively reviewed 1296 patients admitted to their ICU for a wide variety of diseases and over a time span of 5 years [4]. They found that within the first $24 \mathrm{~h}$ of treatment, 549 patients $(42.4 \%)$ fulfilled all „major“-criteria for the applicability of dynamic preload indicators (sedation, absence of arrhythmias and controlled ventilation with a tidal volume above $8 \mathrm{ml} / \mathrm{kg}$ ). This proportion was evenly distributed among sepsis (37\%), post cardiac arrest (39\%) or postoperative $(33 \%)$ patients, with a slightly higher proportion $(51 \%)$ in patients with polytrauma. Notably, more than half of the patients in this review were ventilated with tidal volumes $>8 \mathrm{ml} / \mathrm{kg}$, suggesting that the proportion of patients in which dynamic preload indicators were applicable would be even lower if concepts of lung-protective ventilation had been used more frequently. Moreover, in patients fulfilling the "major" criteria, there was a high prevalence of "minor" limiting factors that could further hamper the reliability of ventilatory-induced variations in stroke volume: lack of an arterial catheter, use of vasopressors and PEEP levels higher than $10 \mathrm{cmH}_{2} \mathrm{O}$. While being criticized for the inappropriate use of permissive factors [5], the study demonstrates that the appropriate use of dynamic preload indicators in the ICU requires a high level of expertise and suspicion concerning the existence of possible limitations to their use.

In the June issue of the Journal of Clinical Monitoring and Computing, Dr. Vistisen and colleagues highlight the failure of the variation in the pre-ejection period (PEP) following deep breathing to predict the hemodynamic response to controlled haemorrhage [6]. The present clinical report may lead to the implication that this marker of hypovolemia should be overlooked in cases of spontaneous ventilation. Interestingly, it might seem peculiar that the authors found that it was impossible to demonstrate such a predictive value. On a first reading, there are several open questions arising from the study of Dr. Vistisen et al.; perhaps the most important is the reason why the cyclic change in the PEP $(\triangle \mathrm{PEP})$ was not able to predict the stroke volume responses to controlled haemorrhage. Indeed, it might seem strange that at the same time, the authors demonstrate that whatever the deep breathing frequency, this maneuver induces significant characteristic fluctuations in the PEP values. Although, we may speculate that a noisy pulse plethysmographic signal may make an accurate assessment of the $\triangle$ PEP difficult [7]; in this regard the methodology used by the authors is perfect. In short, the message from this study is the lack of the ability of the $\triangle$ PEP measurements to assess a preload dependency state and to guide fluid therapy in spontaneously breathing patients. However, the present results should be interpreted with caution as various methodological improvements could significantly increase the reliability of this approach combining deep breath and systolic time interval measurements.

In the August issue of the Journal, the same authors, $D r$. Vistisen and colleagues proposed the idea that the occurrence of an extra systole could be a convenient preload mechanism in animals ventilated with very low tidal volume $(<6 \mathrm{ml} / \mathrm{kg})$. In this regard, they used a paced porcine hemorrhagic model, where they investigated the hemodynamic effects of the post-ectopic beat versus the preceding normal sinus beat to predict fluid responsiveness [8]. Studying as usual the variation in the pre-ejection period (PEP), the authors demonstrated that the changes induced by a ventricular extra systole on PEP (derived from the arterial pressure curve or from the Pleth curve) was able to predict stroke volume response to re-transfusion with 70-75\% sensitivity and specificity and reasonable ROC areas around 0.8 . Interestingly, in the present study the authors found that pulse pressure variation related to ventricular extra systole poorly predict fluid responsiveness. They adequately explained the present finding by the fact that pulse pressure is mainly affected by the declining diastolic pressure following the compensatory pause. However, as a secondary end-point the authors reported also a curious result as they found that variables derived from supra-ventricular extra systoles were not useful for fluid responsiveness prediction, making the hypothesised physiologic mechanism unclear regarding systolic time intervals markers [8].

With another report on the impact of BCP on blood circulation in patients undergoing elective arthroscopic shoulder surgery, Zhu and colleagues reported in the edition of December, a very nice study on plethysmographic (PPG) waveform changes analysis induced by BCP under general anaesthesia [9]. The authors reported how a noninvasive and continuous technique like a PPG signal recorded from a commercial pulse oximeter is able to monitor blood circulation of patients undergoing BCP. Twenty-nine adults undergoing shoulder surgery were monitored before and after beach chair positioning with electrocardiogram, intermittent blood pressure, and photoplethysmograph via finger pulse oximeter. Frequency-domain analysis (Fast Fourier Transform) showed that respiration-associated oscillations of the PPG increased with beach chair positioning (decrease in venous return) in the absence of vasoactive agents with a greater consistency than simultaneously measured changes in BP and HR [9]. The present results are in line with the several studies 
having shown that changes in respiration associated waveform oscillations of PPG during positive pressure ventilation are valuable markers of blood volume status [7]. However, the change in posture for patients induced fewer changes in the magnitude of oscillations of PPG related to respiration when patients were under phenylephrine, suggesting by the way, that a blood venous recruitment may decrease the impact of this position on patient hemodynamics. As usual, the present study highlights the redundant clinical finding consisting in significant changes in haemodynamic status not detected by heart rate and blood pressure variations.

\section{Cardiac output monitoring}

During 2014 the Journal featured several articles on cardiac output monitoring. Safe and easy to use cardiac output monitoring remains one of the "yet to be achieved" goals of clinical monitoring. Available techniques range from indicator methods such as Fick and thermodilution, through Doppler ultrasound applications, to surrogate methods such as pulse contour analysis and bioimpedance. The Year in Review for 2014 featured examples of all of them.

In the February edition Terada and colleagues reported data validating the estimated continuous cardiac output (esCCO) method in children undergoing renal transplant [10]. The esCCO method is noninvasive and simple to use. It measures cardiac output indirectly. Pulse wave transit time (PWTT) is measured from the ECG and pulse oximeter and used to estimate beat to beat stroke volume. The technique is novel and has been recently incorporated into a patient monitor (Nihon Kohden, Tokyo, Japan). The esCCO method has been well studied and validated by Ishihara and colleagues from Japan [11]. The study is unusual because densitometry using indocyanine green dye dilution was used as the reference cardiac output method. Perioperative readings were limited to just two, during and after surgery, and thus trending ability could not be assessed. Patient numbers were limited to 7 children and 10 adults, so the power of the study was poor. In the paediatric cohort the percentage error against dye densitometry was $34 \%$, which is just above the benchmark of $<30 \%$ for interchangeability, so results were promising [12]. In adults the results were less good with a percentage error of $43 \%$. It was assumed that dye densitometry had a similar precision as single bolus thermodilution the reference standard to which most of the current statistical benchmarks in cardiac output validation are set.

Wong and colleagues in the same edition provided a more clinical report of cardiac output monitoring being used to study the progress of children admitted to a hospital for treatment of acute asthma [13]. The cardiac output monitor used was the Aesculon, or portable version the Icon (Cardiotronic Inc., La Jolla, CA, USA) a hybrid bioimpedance device. Rather than using the differential of the transthoracic bioimpedance signal $(\mathrm{dZ} / \mathrm{dt})$ to derive stroke volume the Aesculon uses the second derivative. A study that used an animal flow probe model to investigate the bioimpedance method showed that the second differential of the bioimpedance waveform, or acceleration index (ACI), was better correlated to equivalent aortic flow probe variables than was cardiac output $(n=16$ dogs $)$ [14], providing some rational for the Aesculon's new approach to bioimpedance signal analysis. Wong and colleagues studied 35 children, 11 of whom needed prolonged critical asthma therapy. From initial high levels their cardiac output readings returned to normal levels following treatment. The trending of the haemodynamic variables in the 11 children who needed more intensive therapy was impressive (Fig. 2). These authors were also able to relate cardiac output increases to stress patterns seen on the ECG in the more severe cases of asthma and suggested cardiac output monitoring may be a better indicator of stress cardiomyopathy than traditional heart rate and blood pressure changes.

USCOM-Window to the Circulation by Critchley and Huang also in the February edition outlined how hand held external Doppler can be used intraoperatively to monitor cardiac output [15]. These authors based their report on a single case of an elderly man having robotic surgery for radical cystectomy. Their account provides a comprehensive review of using suprasternal Doppler (USCOM Ltd., Sydney, Australia) intraoperatively. Compared to other indirect methods of measuring cardiac output such as pulse contour analysis, it is difficult to argue against Doppler tracking changes in stroke volume and cardiac output reliably. However, Doppler is a very different technology to use requiring a significant degree of user skill because probes have to be manually focused to obtain optimum scan data. Thus, the flow data obtained is only as good as the operator who collects it. In Window to the Circulation USCOM cardiac output readings were verified against a second oesophageal Doppler method and good a correlation between methods of $r=0.92$ was found (Fig. 5). The case itself demonstrates how significant changes in haemodynamic status that could not be detected from heart rate and blood pressure alone were detected by the USCOM and displayed on its trend data screen. These flow changes were related to intravenous fluid management. The thorax is rich in flow signals other than the aortic outflow signal from which cardiac output is derived. However, advancing age and aortic unfolding can make the aortic outflow signal difficult to detect and this point is demonstrated by the case. The different types of signal arising from within the thorax are also illustrated. 
Another aspect to measure cardiac output was suggested in the August Issue by Ishihara and colleagues who used a new non-invasive continuous cardiac output (CCO) monitoring system called estimated continuous cardiac output (esCCO) [16]. In the esCCO system, $\mathrm{CCO}$ is derived by means of a conventional electrocardiogram (ECG) monitor, peripheral pulse oximetry $\left(\mathrm{SpO}_{2}\right)$ system, and arterial pulse pressure [16]. The esCCO system uses a mathematical equation to estimate stroke volume (SV) by derivation from pulse wave transit time (PWTT); defined as the sum of the pre-ejection period and the time taken for pulse wave to travel from the ascending aorta to the $\mathrm{SpO}_{2}$ probe site. However, because changes in systemic vascular resistance (SVR) affect CCO measurement system with arterial waveform analysis [17], in the present report, the authors analysed the impact of SVR on the esCCO system. Indeed, there have been no clinical studies on the esCCO system concerning CCO estimation during changes in SVR or on the impact of the time component of PWTT on the estimation of $\mathrm{CO}$. In the present paper, the authors reported two retrospective analyses on two patients monitored with both a CCO monitor derived data from a flow directional pulmonary artery catheter (Swan-Ganz CCOmbo CCO/SVO2, Baxter Healthcare, Edwards Critical Care Division, Irvine, CA) and the esCCO system device. Inducing pharmacological changes in systemic vascular resistance, the authors demonstrate a close relationship between each component of PWTT and SV during changes in SVR [16]. There are however, several limitations in this report, in addition to a very small sample size. And the principal one precluding the use of the present concept in the clinical setting is the potential mathematical coupling existing between systolic time intervals, SV and SVR.

In the issue of August, Saugel and colleagues investigated a new non-invasive device able to measure cardiac output on a new basis [18]. Using a sensor that is placed over the radial artery, radial artery applanation tonometry (AT) (Tensys Medical Inc., San Diego, CA, USA) samples arterial pressure (AP) data at $160 \mathrm{~Hz}$ and generates an AP waveform closely matching that obtained by arterial catheterization [18]. On these AT derived pressure data, Saugel et al. applied an autocalibrating algorithm for the calculation of $\mathrm{CO}$ that is based on the analysis of a nonlinear mathematical model using 11 physiological input vectors: gender, age, height, body weight, systolic, diastolic and mean AP, pulse pressure $(\mathrm{mmHg})$, beat-to-beat interval, maximal slope within systole, dP/dtmax, and systolic area. CO-values derived from this algorithm showed a clinically acceptable level of agreement and a percentage error of $23 \%$ when compared to simultaneously obtained $\mathrm{CO}$ measured with pulse contour analysis that was calibrated with transpulmonary thermodilution immediately before the recording intervals. The study of Saugel et al. demonstrates that pressure signals derived from radial AT can be used to compute CO. As a nonlinear analysis of the complete AP curve underlies the mathematical algorithm, this new CO-measurement technology is critically dependent upon an optimal AP waveform signal. Therefore, in this proof-of-concept pilot study, data for the validation of AT-derived CO-measurements were carefully selected by Saugel et al. to identify the most accurate AP signals. The accuracy of this new technology in unselected patients still remains to be investigated.

In the December edition Mattie and colleagues presented data validating a novel adaptation of the Fick method using measurement of carbon dioxide [19]. The technique was first described by Kim et al. in the $1960 \mathrm{~s}$ [20]. In ventilated patients following cardiac surgery prolonged expiratory phases (i.e. $15 \mathrm{~s}$ ) were introduced using a flow restrictor placed in the expiratory limb of the patient ventilation circuit. Carbon dioxide rebreathing had previously been used, but utilizing a slightly different method, to measure cardiac output in the 1990 s by a device called the NICO [21]. However, this system was too unstable because it required regular breathing patterns to become widely adopted into clinical practice. The technical aspects of Kim's method are of interest. However, the authors present their data in an overelaborate way and seem to hide the truth that the system is not sufficiently accurate to be adopted clinically. There was no need for them to divide patients into male and female groups. They also avoided stating the true percentage error which is between 40-45\% which is well above the benchmark of $<30 \%$ for interchangeability [12]. Thermodilution was used as the reference method. The study also lacks any data on trending performance. The method may have excessive time delays that would make trending impractical. However, the authors' regression plot (Fig. 3.) of $\mathrm{CO}(\mathrm{k})$ versus $\mathrm{CO}(\mathrm{td})$ does suggest good concordance as cardiac output increases and suggests good trending ability. Whether the method can be refined to provide more reliable and accurate readings in the future only time will tell.

\section{Cardiac function}

In the December edition Fayssoil and colleagues presented a small albeit interesting study which looked at right ventricular function in patients suffering from Pompe disease, an uncommon glycogen storage disease [22]. Probably due to the low incidence of the disease, patient numbers were limited to 10 adults with late onset Pompe disease and seven control patients, so the power of the study was poor. While left ventricular function has been studied before and found to be unaltered in adult Pompe patients [23], this is 
the first report on right ventricular function (RVF) in these patients. RV dysfunction is of importance since it may impair outcome, as it does in heart failure. RVF was evaluated by 2D transthoracic echocardiography using Doppler (including tissue Doppler) and tricuspid annular plane systolic excursion (TAPSE) measurements. As with left ventricular function, RVF was not altered. It has to be mentioned, however, that RVF is difficult to assess due to the complex structure and asymmetrical, crescent shape of the right ventricle, especially by transthoracic 2D echocardiography. More sophisticated methods (e.g. 3D transesophageal echo) and larger sample sizes might thus come to different conclusions.

\section{References}

1. Jo YY, Jung WS, Kim HS, Chang YJ, Kwak HJ. Prediction of hypotension in the beach chair position during shoulder arthroscopy using pre-operative hemodynamic variables. J Clin Monit Comput. 2014;28(2):173-8.

2. Rex S, Brose S, Metzelder S, Huneke R, Schalte G, Autschbach $\mathrm{R}$, Rossaint R, Buhre W. Prediction of fluid responsiveness in patients during cardiac surgery. Br J Anaesth. 2004;93(6):782-8.

3. Maguire S, Rinehart J, Vakharia S, Cannesson M. Technical communication: respiratory variation in pulse pressure and plethysmographic waveforms: intraoperative applicability in a North American academic center. Anesth Analg. 2011;112(1): 94-6.

4. Benes J, Simanova A, Tovarnicka T, Sevcikova S, Kletecka J, Zatloukal J, Pradl R, Chytra I, Kasal E. Continuous non-invasive monitoring improves blood pressure stability in upright position: randomized controlled trial. J Clin Monit Comput. 2015;29(1): 11-7.

5. Pinsky MR. It is amazing what you can see if you look. J Clin Monit Comput. 2014;28(3):221-2. doi:10.1007/s10877-0139525-7.

6. Vistisen ST, Juhl-Olsen P, Frederiksen CA, Kirkegaard H. Variations in the pre-ejection period induced by deep breathing do not predict the hemodynamic response to early haemorrhage in healthy volunteers. J Clin Monit Comput. 2014;28(3):233-41.

7. Bendjelid K. The pulse oximetry plethysmographic curve revisited. Curr Opin Crit Care. 2008;14(3):348-53.

8. Vistisen ST, Andersen KK, Frederiksen CA, Kirkegaard H. Variations in the pre-ejection period induced by ventricular extra systoles may be feasible to predict fluid responsiveness. J Clin Monit Comput. 2014;28(4):341-9.

9. Zhu R, Atteya G, Shelley KH, Silverman DG, Alian AA. Analysis of plethysmographic waveform changes induced by beach chair positioning under general anesthesia. J Clin Monit Comput. 2014;28(6):591-6.

10. Terada T, Maemura Y, Yoshida A, Muto R, Ochiai R. Evaluation of the estimated continuous cardiac output monitoring system in adults and children undergoing kidney transplant surgery: a pilot study. J Clin Monit Comput. 2014;28(1):95-9.

11. Ishihara H, Okawa H, Tanabe K, Tsubo T, Sugo Y, Akiyama T, Takeda S. A new non-invasive continuous cardiac output trend solely utilizing routine cardiovascular monitors. J Clin Monit Comput. 2004;18(5-6):313-20.

12. Critchley LA, Critchley JA. A meta-analysis of studies using bias and precision statistics to compare cardiac output measurement techniques. J Clin Monit Comput. 1999;15(2):85-91.

13. Wong J, Dorney K, Hannon M, Steil GM. Cardiac output assessed by non-invasive monitoring is associated with ECG changes in children with critical asthma. J Clin Monit Comput. 2014;28(1):75-82.

14. Peng ZY, Critchley LA, Fok BS, James AE. Evaluation of impedance based indices of cardiac contractility in dogs. J Clin Monit Comput. 2004;18(2):103-9.

15. Critchley LA, Huang L. USCOM-window to the circulation: utility of supra-sternal Doppler in an elderly anaesthetized patient for a robotic cystectomy. $\mathrm{J}$ Clin Monit Comput. 2014;28(1):83-93.

16. Ishihara H, Tsutsui M. Impact of changes in systemic vascular resistance on a novel non-invasive continuous cardiac output measurement system based on pulse wave transit time: a report of two cases. J Clin Monit Comput. 2014;28(4):423-7.

17. Biais M, Cottenceau V, Petit L, Masson F, Cochard JF, Sztark F. Impact of norepinephrine on the relationship between pleth variability index and pulse pressure variations in ICU adult patients. Crit Care. 2011;15(4):R168.

18. Saugel B, Meidert AS, Langwieser N, Wagner JY, Fassio F, Hapfelmeier A, Prechtl LM, Huber W, Schmid RM, Godje O. An autocalibrating algorithm for non-invasive cardiac output determination based on the analysis of an arterial pressure waveform recorded with radial artery applanation tonometry: a proof of concept pilot analysis. J Clin Monit Comput. 2014;28(4):357-62.

19. Mattei A, Schena E, Cecchini S, Proscia P, Saccomandi P, Silvestri S, Carassiti M. Non-invasive cardiac output evaluation in postoperative cardiac surgery patients, using a new prolonged expiration-based technique. J Clin Monit Comput. 2014; 28(6):625-32.

20. Kim TS, Rahn H, Farhi LE. Estimation of true venous and arterial PCO2 by gas analysis of a single breath. J Appl Physiol. 1966;21(4): 1338-44.

21. Haryadi DG, Orr JA, Kuck K, McJames S, Westenskow DR. Partial CO2 rebreathing indirect Fick technique for non-invasive measurement of cardiac output. J Clin Monit Comput. 2000; 16(5-6):361-74.

22. Fayssoil A, Nardi O, Annane D, Orlikowski D. Right ventricular function in late-onset Pompe disease. J Clin Monit Comput. 2014;28(4):419-21. doi:10.1007/s10877-014-9551-0.

23. Soliman OI, van der Beek NA, van Doorn PA, Vletter WB, Nemes A, Van Dalen BM, ten Cate FJ, van der Ploeg AT, Geleijnse ML. Cardiac involvement in adults with Pompe disease. J Intern Med. 2008;264(4):333-9. doi:10.1111/j.1365-2796. 2008.01966.x. 